\title{
XXII.
}

\section{Ueber die Entstehung der Hippursäure nach dem Genusse von Benzoësäure.}

Von Dr. W. Kühne und W. Hallwachs.

Die Entdeckung von Wöhler und Keller, dass der Harn nach dem Genusse von Benzoësäure Hippursäure enthält, ist eine bis jetzt noch unerklärte Thatsache. Die leichte Zersetzbarkeit der Hippursäure unter Einwirkung concentrirter Säuren oder Alkalien in Benzoësäure und Glycin liess vermuthen, dass die Benzoësäure im Körper das Letztere als Paarling vorfinde und sich damit unter Austritt von 2 Aeq. Wasser verbinde. Man mag über die Constitution der Hippursäure eine Ansicht haben, welche man will, es steht fest, dass es bis jetzt nur durch Berücksichtigung der genannten Zersetzung gelungen ist, aus einer Glycin- und Benzoylverbindung Hippursäure künstlich darzustellen; und wenn auch jene hier gemeinte Entstehung derselben bei der wechselseitigen Zersetzung des Benzoylehlorürs und der Zinkverbindung des Glycins keineswegs dem im Organismus wahrscheinlich vor sich gehenden Processe analog zu sein scheint, so kann man doch mit gutem Grund annehmen, dass die Bestandtheile des Körpers, welche vermuthlich Glycin enthalten, hierbei eine wesentliche Rolle spielen. Wir sehen ja auch nach dem Genusse anderer Verbindungen, z. B. der Salicyl- und Toluylsäure mit Glycin gepaarte Säuren in dem Harn auftreten, und es findet mithin die Annahme einer einfachen Verbindung unter Austritt von Wasser hierin eine neue Stütze.

Das Auftreten von Glycin im Körper kann man wohl mit Sicherheit der rückgängigen Metamorphose der leimgebenden Gewebe zuschreiben, doch bleibt es immerhin noch unklar, ob eine solche, ähnlich der auch ausserhalb des Thierleibes beobachteten Zersetzung des Bindegewebes, in allen Blutgefässen oder nur in denen der Leber, deren Secret bekanntlich als wesentlichen Bestandtheil Glycin 


\section{7}

enthält, vor sich geht. Wir haben nun in der nachstehenden Untersuchung den ort, so wie speciell die Secrete kennen zu lernen versucht, welche der eingeführten Benzoësäure das Glycin als Paarling zuführen konnten, und suchten zu dem Ende folgende Fragen durch Experimente zu beantworten.

I. Wird die genossene Benzoësäure nach der Resorption durch das Blut an und für sich in Hippursäure umgewandelt, und findet folglich dieser Process im ganzen Blutgefässsystem Statt?

Oder

II. Wird die Benzoësäure nur durch Einwirkung der Galle, also entweder schon im Darmrohr oder innerhalb der Gefässe der Leber in Hippursäure umgewandelt?

Zur Entscheidung der ersten Frage kann man keinen anderen Weg einschlagen, als den der directen Zuführung von Benzoësäure ins Blut, also den der Injectionen.

Ehe wir indessen zur näheren Beschreibung der angestellten Versuche übergehen, muss hier noch Einiges über die Prüfung des Harns auf Hippursäure oder Benzoësäure bemerkt werden. Dieselbe wurde bei allen Versuchen auf folgende Weise ausgeführt. Der möglichst frische, häufig durch Drücken aus der Blase der Thiere gewonnene Harn wurde, falls er stark sauer war, mit Natron neutralisirt, auf dem Wasserbade zur Trockne abgedampft; der Rückstand mit $85^{\circ}$ starken Alkohol bei etwa $50^{\circ}$ G. ausgezogen, das alkoholische Extract unter Zusatz von Oxalsäure bei gelinder Temperatur bis zur Trockne abgedampft, die Masse hierauf mit Glaspulver oder mit durch Salzsäure gereinigtem Sand zerrieben und so mit Aether ausgezogen.

Die ätherische Lösung enthält dann neben ziemlich bedeutenden Mengen von Oxalsäure die gesuchte Hippursäure. Da sich immer Oxalsäure mit in dem Aether auflöst, dieselbe auch in absolutem Aether weit leichter löslich ist als meist angegeben wird, so ist es unmöglich in dem Rückstande des ätherischen Auszuges kleine Mengen yon Hippursäure neben der Oxalsäure mit Sicherheit zł bestimmen. Man muss deshalb den Rückstand noch mit 
wenig Kalkmilch auskochen, filtriren, das Filtrat bei gelinder Temperatur bis zur geeigneten Concentration abdampfen und mit Salzsäure versetzen. Ist Hippursäure vorhanden, so scheidet sie sich dann meist ziemlich rein ab und kann dann durch Prüfung der Krystalform und Erhitzen in einem Röhrchen an dem characteristischen Auftreten von Benzonitril und der Sublimation von Benzoësäure leicht erkannt werden. Auf dieselbe Weise kann dann auch etwa vorhandene. Benzoësäure dargestellt und nachgewiesen werden.

Injections versuche.

Nur Fleischfresser sind zu diesen Versuchen tauglich, da der Harn aller Pflanzenfresser an und für sich schon Hippursäure enthält.

Dass der Harn von Hunden, die wir zu allen unseren Versuchen benutzten, bei gewöhnlicher Fütterung mit Brod, Milch oder. Fleisch niemals Hippursäure enthält, liess sich vermuthen, und wir vermochten wenigstens durch obige Methode nie dieselbe darin nachzuweisen.

Injectionen von Benzoësäure in die Venen sind stets mit einigen Schwierigkeiten verknüpft, was seinen Grund in der geringen. Löslichkeit dieser Säure hat. Wir suchten uns dadurch zu helfen, dass wir eine gesättigte Lösung derselben von $40^{\circ} \mathrm{C}$. in Wasser herstellten und daron jedesmal bis zu $50 \mathrm{Ccm}$. und darüber injicirten. Auf die Weise gelingt es dann wohl bis zu $0,5 \mathrm{Gr}$. davon ins Blut zu bringen, welche Menge in den Magen gebracht, wie wir uns durch Versuche überzeugten, stets gut nachweisbare Quantitäten von Hippursäure im Harn lieferte.

Alkoholische lösungen schienen uns wegen der dabei eintretenden Trunkenheit und mithin erhöhten Respiration ungeeignet.

Bei etwa 20 Versuchen, die wir so anstellten und wo wir hintereinander in der Regel bei demselben Thiere beide Jugularund Cruralvenen zur Injection benutzen, fanden wir in dem im Verlauf von 24 bis zu 30 Stunden nach der Operation gesammelten Harn niemals Hippursäure, aber befremdender Weise auch keine Benzoësäure. Es war daher denkbar, dass die Benzoësäure, wenn sie wirklich im Blut nicht in Hippursäure umgewandelt wurde einfach durch die Respiration oder Perspiration eine vollkommene 


\section{9}

Zersetzung erlitt. Indessen sind wir nicht geneigt diese Versuche a]s beweisend anzusehen, da bei Injectionen von erwärmten Flüssigkeiten, die beim sinken der Temperatụ rasch krystallisiren, leicht Blufstockungen durch Gerinnselbildungen in den Gapillaren stattfinden, wodurch dann das Resultat der Harnuntersuchung unter solchen gewiss nicht normalen Bedingungen als irrelevant erscheinen musste. Diese durch kleine Benzoësäurekrystalle entstehenden Blutgerinnsel zeigten sich sehr deutlich, als wir, um grössere Dosen dieser Säure injiciren zu können, dieselbe in sehr fein vertheiltem Zustande mit Wasser oder Gummilösung angerieben ins Blut brachten. Die. Thiere erlagen danach schon in 10 Stunden unter den Erscheinungen der Athemnoth. Bei der gleich nach dem Tode angestellten Section zeigten sich die sonst ganz gleichmässig hellroth gefärbten Lungen der Hunde mit grossen dunkelrothen Flecken besetzt. "An solchen Stellen waren dieselben luftleer, hart und knisterten nicht beim Drücken; über die Schnittfläche ergoss sich meist ein wässeriges Exsudat, und unter dem Mikrosḳop liessen sich stets in den feinsten Capillaren neben Faserstoffmassen kleine Benzoësäurekrystalle erkennen. Die Luftröhre fand sich stets mit einem zăhen Schaum erfüllt. Da also auch diese Methode der Benzoësäurezuführung ungeeignet war, so blieb uns nichts Anderes übrig als die leicht löslichen benzoësauren Alkalien. Seltsamer Weise geht benzoësaures Natron ohne Zersetzung, d. h. nicht als kohlensaures Natron in den Harn über. Man kann ohne Schaden bis zu 3 Gr. benzoësaures Natron verschlucken; der Harn wird hierdurch nicht alkalisch, wie sonst nach dem Genusse der Alkaliverbindungen mit den organischen Säuren, die bekanntlich, so weit dies untersucht, als kohlensaure Salze in dem Harn wieder erscheinen, und bei der Untersuchung zeigte sich, dass alles benzoësaure Natron als hippursaures Natron in den Harn übergegangen war. Nach Feststellung dieser Thatsachen haben wir kein Bedenken getragen, auch Lösungen von benzoësaurem Natron in die Venen $\mathrm{zu}$ injiciren. Natürlich hat man dafür zu sorgen, dass kein Ueberschuss an Alkali vorhanden ist, was schädlich auf die Gefässwandungen wirken kann. Bei einer Injection von $50 \mathrm{Gcm}$. konnten wir nun leicht bis zu 4 . Gr. Benzoësäure ins Blut befördern, 


\section{0}

wonach dann die Untersuchung des Harns sehr entschiedene Resultate liefern musste.

Nach solchen Injectionen konnten wir nun in der That beträchtliche Mengen dieses Salzes im Harn wieder finden, woraus hervorgeht, dass auch das benzoësaure Natron, selbst ohne vorhergegangene Ueberführung in hippursaures Natron sehr kräftig den oxydirenden Einwirkungen der Respirationsprocesse widersteht. Der Harn wurde hierbei ebenfalls nie alkalisch und blieb unter sonst normalen Verhältnissen immer sauer. Neben der aus demselben erhaltenen Benzoësäure fanden sich indessen immer einige feine Nadeln, die allenfalls Hippursäure sein konnten. Jedoch war ihre Menge so gering, dass sie nicht von den Benzoësäurekrystallen gesondert werden konnten und daher nicht genau zu prüfen waren.

Angenommen nun, dass also dennoch nach Benzoësäureinjectionen der Harn Spuren von Hippursäure aufweist, so wäre dies noch keineswegs beweisend für die Entstehung derselben im Blut, da doch höchst wahrscheinlich nach der Injection auch die Secrete, welche in das Verdauungsrohr gelangen, die injicirte Masse mit dahinfuhren, und so die Untersuchung des Harns kein ganz exactes Resultat liefern kann.

Auch würde ja ein, wenn auch nur geringer Theil der injicirten Lösung nach seinem Durchgang durch die Darmcapillaren mit der Pfortader in die Leber befördert werden, und falls hier der Ort der in Rede stehenden Umwandlung wäre, eine geringe Quantität Hippursäure entstehen können. Es wäre aber auch noch der Fall denkbar, dass geringe Massen des benzoësauren Natrons mit der Galle selbst in den Darm überträten, und nachdem dieselben hier der vermuthlichen Einwirkung dieses Secrets ausgesetzt wären, als hippursaures Natron wieder aufgesogen würden. Alle diese Annahmen konnten gemacht werden, um das etwaige Auftreten geringer Mengen von Hippursäure nach Injection von benzoësaurem Natron zu erklären. Jedoch wiederholen wir hier, dass es uns unmöglich war die Natur dieser kleinen zwischen der Benzoësäure auftretenden Krystalle genauer zu ermitteln. Sämmtliche besprochenen Injectionsversuche würden übrigens begreiflicher Weise 
eine nur geringe Beweiskraft für die Lösung der oben angeführten ersten Frage haben, wenn sie nicht durch die Ergebnisse der zur Lösung unserer zweiten Frage angestellten Versuche unterstützt würden. Bei der schwierigen Feststellung negativer Resultate haben wir, um ganz sicher zu gehen, namentlich die Injectionsversuche sehr häufig wiederholt. Wir halten es aber für überflüssig, hier eine unvermeidlich monotone Aufzählung derselben folgen zu lassen.

Da die Gegenwart von Glycin mit Sicherheit nur in der Galle nachgewiesen ist, so lag es nahe, die Hippursäurebildung der Einwirkung des glycocholsauren Natrons zuzuschreiben. Um hierüber Aufklärung zu erlangen, musste man also den Thieren Benzoësäure eingeben und auf irgend eine Weise den Zufluss oder die Einwirkung der Galle abschneiden. Dies geschah zunächst durch Anlegung von Gallenfisteln.

1. Versuche an Hunden mit Gallenfisteln.

Bei Anlegung dieser Fisteln wurde nach der Arnoldschen Methode verfahren. Wir können aus Erfahrung bestätigen, dass es nur durch Anwendung der von Arnold angegebenen Canüle gelingt, eine dauernde und sich nicht verschliessende Fistel herzustellen. Die von Bidder und Schmidt angegebenen Canülen lassen sich nur schwierig an der Gallenblase befestigen, und die Oeffnung derselben ist zu klein, um der Galle einen ungehinderten Abfluss zu verschaffen.

Obgleich wir sehr viele Gallenfisteln anlegten, wollte es uns übrigens doch nie gelingen, den Rand der Canüle zwischen Haut und Bauchmuskeln einzuheilen. Dieselbe blieb allerdings 8-14 Tage sitzen, wurde aber dann durch die stark sich aufwulstenden Wundränder hervorgedrängt, so dass die Fäden, womit sie eingenäht war, nachgaben.- Später von Neuem Canülen einzubringen, schien uns nicht rathsam, zumal die Fistel immer eine gehörige Weite behielt.

Da es bei unsern Versuchen nöthig war, dass die Galle durchaus nicht in den Verdauungscanal gelangte, so mussten die Hunde durch Hochbinden des Kopfes an dem Auflecken derselben verhindert werden. Hierauf wurde den Thieren mittelst der Schlund- 
sonde von 1 bis zu 4 Gr. Benzoësäure, entweder als Emulsion mit Wasser oder in Natron gelöst, in den Magen gespritzt, und der in der Regel nach 12 Stunden gelassene Harn untersucht. Derselbe enthielt stets Hippursäure und keine Benzoësäure, ein Beweis also, dass die Hippursäurebildung nicht von der in das Duodenum fliessenden Galle abhängig ist. Zum Ueberfluss wurde noch ein Controllversuch gemacht, der darin bestand, dass wir einen Hund knurze Zeit nach dem Genusse von 3 Grm. Benzoësäure tödteten und den Inhalt des ganzen Dünndarms untersuchten. Die dicke schleimige Masse wurde auf dem Wasserbade zur Trockne gebracht, mit Alkohol ausgezogen und wie oben angegeben; auf Hippursäure geprïft. Wir fanden dieselbe darin nicht, wohl aber Spuren von Benzoësäure. Durch diesen Gègenversuch war also vollkommen bewiesen, dass falls überhaupt die Galle der genossenen Benzoësäure das nöthige Glycin lieferte, doch keineswegs auf den allerersten Wegen (im Duodenum) die Hippursäure gebildet wird: Deshalb wurde es wahrscheinlich, dass da, wo das Glycin voraussichtlich entsteht, in dem Gewebe der Leber nämlich, auch gleichzeitig Benzoësäure in Hippursäure umgewandelt wird. Bevor wir jedoch zu den hieriber entscheidenden Versuchen übergehen, sei es uns erlaubt, noch Einiges über die Erscheinungen, welche Thiere mit Gallenfisteln bieten, zu bemerken.

Gewöhnlich lecken die. Thiere unaufhörlich an der Fistelöffnung, so dass gar keine Galle für sie verloren geht. Deshalb sieht man an denselben, nachdem die ersten Folgen der Operation überstanden sind, auch durchaus keine krankhaften Erscheinungen. Sie fressen mit gutem Appetit auch die fetteste Nahrung, und die Beschaffenheit der Excremente bleibt normal. Anders verhält es sich aber, wem man die Thiere auf irgend eine Weise längere Zeit an dem Auflecken der Galle verhindert. Unsere Hunde zeigten unter diesen Umständen durchaus keine übermässig grosse GeCrässigkeit, fette Nahrung, wie Milch etc. verschmähten sie gänzlich; die Excremente wurden dünn und übelriechend. Dabei wurden die Thiere sehr matt und träge und magerten ausserordentlich ab. Auch konnten wir stets jenes auffallende Ausfallen der Haare beobachten. Zwei Hunde mit Gallenfisteln, die wir etwa 4 Wochen am 


\section{3}

Leben zu erhalten vermochten, starben an Erstickung. Bei der Section zeigten sỉch Luftröhre, Kehlkopf und Rachenhöhle mit dikken lamellösen Schleimmassen bedeckt. Es ist möglich, dass zu dieser Todesart die ungünstige kalte Jahreszeit beigetragen. . Was den Ort der Operation anbetriff, so. war der Fundus der Gallenblase durch ein festes fibröses Stroma mit den Bauchdecken verwachsen. Die Schleimhaut der Gallenblase und der Duct. hepat. normal.

Die Unterbindungsfäden am Duct. choledochus waren in $\mathrm{Ab}$ scessen eingeschlossen, während rings herum die Leber, der Magen und das Duodenum durch feste Narbensubstanz aneinander geheftet waren.

Beim Ablösen dieser Theile von einander zeigte sich namentlich der untere Theil der Leber mit kleinen Eiterheerden durchsetzt. Die Schleimhäute des Magens und Duodenums normal, dagegen die Muscularis durch die Contraction der herumliegenden vernarbten Theile stark verdickt. Eine Wiederherstellung des Duct. choledochus sahen wir nie. Auch die von Kölliker und Heinr. Müller beobachteten perforirenden Magengeschwüre und Arterienincrustationen, hatten wir leider nicht Gelegenhèit zu beobachten. Die Bauchwunde bildete eine grosse. strahlige Narbe.

II. Versuche bei Abschluss des Leberkreislaufs.

Die gerühmte zähe Natur der Katzen veranlasste uns, die Versuche, bei denen das ganze Gefässsystem der Leber abgeschlossen sein sollte, bei diesen Thieren anzustellen. Wir öffneten zu dem Ende bei einer durch Aether betäubten Katze die Bauchhöhle, zogen um den ganzen Hilus der Leber mit einem starken Faden eine Schlinge und unterbanden damit den Duct. choledochus, sowie alle zu- und abführenden Gefässe. Nachdem die Bauchwunde sorgfältig zugenäht, wurden mit der.Schlundsonde einige Grm. Benzoësäure oder benzoësaures Natron mit viel Wasser in den Magen gespritzt. Vorher ward das Thier gut gefüttert und getränkt, um eine möglichst reichliche Harnabsonderung zu bewirken. Dreimal wiederholten wir diesen Versuch, wobei der Tod nach 5, 7 und 10 Stunden erfolgte. Bei der Section hatten wir auch das Glück, die Harnblase strotzend gefüllt $z u$ sehen: Der Inhalt derselben 


\section{4}

reagirte alkalisch und enthielt namentlich in einem Falle grosse Mengen von Benzoësäure, dagegen gar keine Hippursäure. Es schien uns dies die Unmöglichkeit der Hippursäurebildung bei abgeschlossenem Leberkreislauf $\mathrm{zu}$ beweisen. Indessen konnte man immer noch einwenden, dass nach einer so gewaltsamen operation, die ein so wichtiges Organ, wie die Leber plötzlich ausser Thätigkeit setzt, jene zur normalen Hippursäurebildung nöthigen Bedingungen vielleicht so verändert würden, dass dadurch allein schon die Benzoësäure unverändert in den Harn übergehen müsse. Deshalb hielten wir es auch für nöthig, durch Gegenversuche zur völligen Gewissheit zu gelangen. Wenn also innerbalb des Bluts bei Gegenwart von bestimmten Gallenbestandtheilen Benzoësäure zu Hippursäure wird, so musste auch nach Injectionen eines Gemisches von Galle mit Benzoësäure ceteris paribus Hippursäure durch die Nieren ausgeschieden werden.

III. Injectionsversuche mit Benzoësäure und den

Bestandtheilen der Galle.

Man kann ohne Schaden Hunden bis zu $40 \mathrm{Gcm}$. Galle injiciren, wenn dieselbe von andern Hunden, entweder nach dem Tode aus der Gallenblase aufgefangen oder aus einer, Gallenfistel gewonnen wurde. Da indessen auf diese Art immer nur geringe, wenigstens nicht zu mehreren Versuchen ausreichende Mengen zu erhalten waren, so zogen wir es vor, nachdem wir eine Injection von Benzoësäure mit Hundegalle gemacht hatten, dazu Ochsengalle zu verwenden. Injicirt man indessen frische, noch Schleim enthaltende Ochsengalle, so gehen die Thiere unter pyämischen Erscheinungen zu Grunde. Es ist daher nöthig, die frische Ochsengalle zur Trockne abzudampfen und mit absolutem Alkohol auszuziehen, wonach der Schleim ungelöst zurìckbleibt. Diese alkoholische Lösung wurde dann ebenfalls zur Trockne abgedampft und der Rückstand in destillirtem Wasser aufgelöst. Injicirten wir nun etwa $1 \mathrm{Gr}$. an Natron gebundene Benzoësäure und $30 \mathrm{Ccm}$. so gereinigter und in der der ursprünglichen Gallenquantität entsprechenden Menge Wasser gelösten Galle, so enthielt der Harn jedesmal Hippursäure und keine Benzoësäure, wurde aber der Injectionsmasse weniger Galle zugemischt, so erschien im Harn neben Hippursäure auch unver- 
änderte Benzoësäure. Es ist also klar, dass die Hippursäurebildung lediglich von der Anwesenheit der Gallenbestandtheile im Blut abhängig ist, und dass diese Bedingung im Gefässsystem der Leber einzig und allein erfüllt werden kann, lejdet wohl keinen Zweifel. Auch beweisen diese Versuche, dass die Umwandlung der Benzoësäure, wenn nur Gallenbestandtheile vorhanden sind, sehr rasch vor sich geht, da wir nach den angeführten Injectionen selbst nach kurzer Zeit schon Hippursäure im Harn fanden, und es widerlegt sich dadurch auch ein Einwand; den man gegen die Beweiskraft der im Eingange unserer Arbeit besprochenen Versuche machen könnte, nämlich der, dass nach Injectionen von Benzoësäure oder benzoësaurem Natron der Harn deshalb keine Hippursäure enthalten könne, weil die injicirte Masse zu rasch das Blut wieder durch die Nieren verlasse, während nach der Aufnahme von Benzoësäure durch den Magen diese nur allmälig resorbirt werde und sich deshalb den vermuthlichen Einwirkungen, welche die Umwandlung in Hippursäure bewirken, besser aussetzen könne.

Schliesslich injicirten wir noch benzoësaures Natron mit gleichen Mengen von reinem glycocholsaurem Natron.

Der darauf gelassene Harn reagirte alkalisch und brauste nach Säurezusatz, enthielt aber beträchtliche Mengen von hippursaurem Natron. Es scheint also, als wenn hier durch einfache Spaltung der Glycocholsäure hippursaures Natron und cholalsaures Natron entsteht, und dass Letzteres durch die Oxydationsprocesse als kohlensaures Natron mit dem Harn den Körper verlässt.

In der Hoffnung, auch künstlich ausserhalb des Organismus einen ähnlichen Effect zu bewirken, digerirten wir sowohl Galle als auch reines glycocholsaures Natron mit benzoësaurem Natron bei einer Temperatur von $+32^{0} \mathrm{C}$. Indessen hatte sich keine Spur von Hippursäure gebildet und es ist bis jetzt nicht abzusehen, welche Bedingungen im Thierleibe vorhanden sind, deren Nichterfüllung solche künstliche Nachahmungen der physiologischen Processe meist vereitelt.

Injectionen von benzoësaurem Natron und Glycin lieferten ebenfalls hippursäurehaltigen Harn, wonach also eine einfache Ver- 
bindung der Benzoësäure mit Glycin unter Austritt von 2 Aeq. Wasser im Blut sehr leicht möglich zu sein seheint.

Fassen wir jetzt kurz die Resultate unserer Versuche zusammen, so ergiebt sich, dass die Bildung der Hippursäure aus genossener Benzoësäure nicht im Verdauungsrohr noch im Blutgefässsystem im Allgemeinen stattfindet, sondern dass innerhalb der Lebergefässe, also im Blut bei Gegenwart von Gallenbestandtheilen, und zwar durch eine Spaltung der Glycocholsäure aus der Benzoësäure Hippursäure entsteht.

Göttingen, den 9. April 1857.

Die vorstehende Untersuchung wurde mit den Mitteln des physiologischen Instituts in Göttingen ausgeführt. Wir sprechen hier dem Direktor desselben, Herrn Hofrath R. Wa gner, für seine freundliche Unterstützung unsern besten Dank öffentlich aus.

\title{
XXIII.
}

Nachträgliche Bemerkungen.

\section{Zur Metamorphose der Bernsteinsäure.}

\author{
Von Dr. W. Kühne.
}

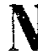

achdem wir in der oben mitgetheilten Untersuchung den Ort, wo die in den Körper eingeführte Benzoësäure in Hippursäure verwandelt wird, sowie die chemischen Verbindungen, durch welche dieselbe bedingt ist, kennen gelernt hatten, bleibt noch Einiges über die mechanischen Verhältnisse zu erörtern übrig.

Wir erwähnten bereits, dass es nicht gelungen sei, ausserhalb des Körpers aus der Benzoësäure durch Digestion mit Galle oder glycocholsaurem Natron künstlich Hippursäure zu bilden, und ich
\end{abstract}

